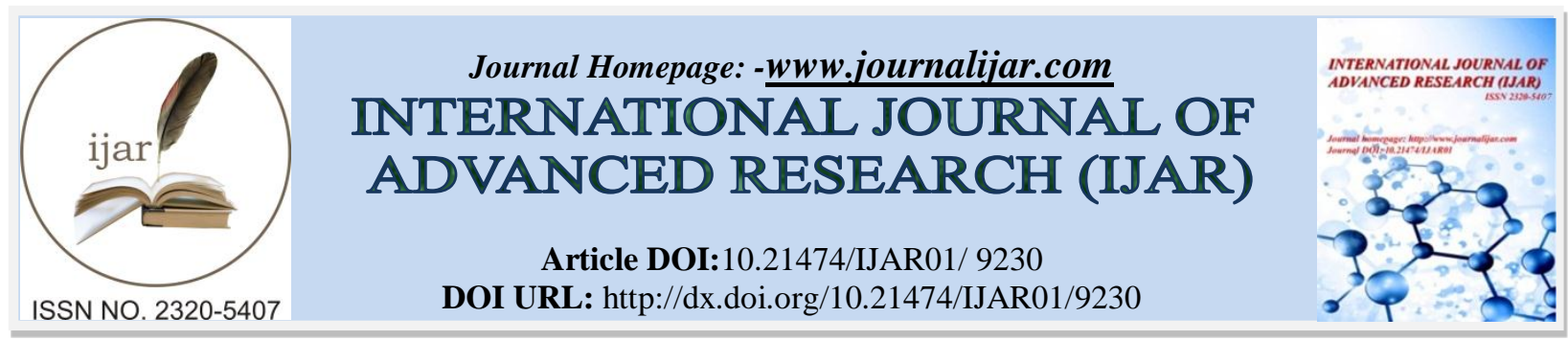

RESEARCH ARTICLE

\title{
« NOVICIAT PEDAGOGIQUE » : PRATIQUES INNOVANTES DE L'ORIENTATION PEDAGOGIQUE EN MILIEU UNIVERSITAIRE MAROCAIN.
}

Fatiha Benmimoun ${ }^{1}$, Mustapha Elmalki ${ }^{2}$, Mohamed Anouar Benaissa ${ }^{3}$ and Abdelhanine Belhaj ${ }^{4}$.

1. Belhaj A., Professeur chercheur à la Faculté des Sciences de l'Education, Université Mohamed V, Rabat, Maroc.

2. Benaissa M.A., Professeur chercheur, équipe : Sciences de l'ingénieur, sécurité et sciences entrepreneuriales (SIMoLab.net), CEDoc : Sciences et Techniques, Université Ibn Tofail, Kénitra, Maroc.

3. Elmalki M., Doctorant (PhD Student), équipe : Recherches Interdisciplinaires pour l'Innovation en Didactiques et en Capital Humain (RIIDCH), laboratoire : Analyse et Évaluation des Systèmes d'Éducation et de Formation (AESEF), CEDoc : Homme, Société et Education, Faculté des Sciences de l'Education, Université Mohamed V, Rabat, Maroc.

4. Benmimoun F., Doctorante (PhD Student), équipe : RIIDCH, laboratoire : AESEF, CEDoc : Homme, Société et Education, Faculté des Sciences de l'Education, Université Mohamed V, Rabat, Maroc.

\section{Manuscript Info}

(...........................

Manuscript History

Received: 06 April 2019

Final Accepted: 08 May 2019

Published: June 2019

Key words:-

Pedagogical orientation, active orientation, professional integration, educational novitiate.

\section{Abstract}

The issue of the introduction day and orientation of new students in Moroccan universities and the reorientation of failed students at the end of the first year requires an examination of the arrangements made to ensure students achieve both academic and professional success. Thus concerns the entire process chain from upstream (High school degree studies) to downstream (Professional integration into workforce), both from the point of view of the actions and practices in place and implemented but also from the point of view of the adaptation of the essential training offered for this combine objective fulfillment. Institutional and political discourse emphasises the importance of "active orientation" at the university as a palliative of failure.

Based on the overview of the specific literature on this topic on the one hand, but also recommendations and suggestions -formulated and debated by the speakers and participants in the first edition of the Scientific Day, organized by us, which was held at the Ibn Tofail University (Kenitra, Morocco) and which echoed in the press ${ }^{1}$, on the theme of the pedagogical orientation and the problem of the professional integration of the young university graduates- of guiding practices, on the other hand. This article focuses on the challenges facing the Moroccan university, namely the increase in global training needs and the desire to train for employment, but also the heavy constraints that weigh on it, given to the reception of a poorly prepared public for the pursuit of higher education. It thus proposes a framework of reflection focused on an oriented pedagogical practices by introducing an innovating device named "educational noviciate".

Copy Right, IJAR, 2019,. All rights reserved.

Corresponding Author:-Fatiha Benmimoun.

Address:-Abdelhanine Belhaj, Professeur chercheur à la Faculté des Sciences de l'Education, Université Mohamed V, Rabat, Maroc. 


\section{Introduction:-}

À l'université, l'accueil des étudiants non ou peu préparés aux exigences de la filière qu'ils ont choisie, ou encore de ceux engagés dans des voies aux débouchés incertains, se traduit généralement soit par un taux d'échec ou même d'abandon important par ces nouveaux bacheliers, soit par des difficultés d'insertion professionnelle en fin de cursus.

Au Maroc, la fin de l'année scolaire 2018 s'est soldée par un taux de réussite de 57\% des bacheliers, marquant ainsi une augmentation de $7 \%$ par rapport à l'année précédente ${ }^{2}$ pour ce qui est des secteurs public et privé réunis. La majorité de ces néo-bacheliers va s'inscrire, faute de mieux, dans les universités marocaines et plus exactement dans des établissements à accès ouvert.

Faute de mieux, parce qu'aux yeux de ces lauréats, l'université reste toujours un choix par défaut eu égard à une école d'ingénieur, de commerce ou à une université étrangère qui, eux, garantissent in fine une meilleure employabilité.

La problématique de l'accueil et de l'orientation des nouveaux étudiants dans les universités marocaines et de la réorientation des étudiants en échec en fin de première année implique donc l'examen des dispositions prises pour assurer aux étudiants un double succès académique et professionnel. Elle concerne par conséquent toute la chaine qui va de l'amont (depuis les études en cycle secondaire) à l'aval (jusqu'à l'insertion professionnelle), tant du point de vue des actions et pratiques préparées et mises en œuvre que du point de vue de l'adaptation de l'offre de formation à ce double objectif.

Dans le discours institutionnel et politique marocain émerge le concept d'« orientation active » à l'université, en tant que palliatif de l'échec et du décrochage. Ce discours renvoie à une histoire et à une tradition nationale assez ancienne dans le domaine de l'orientation. En effet, au Maroc et dans le cadre du système universitaire massifié, où les taux d'abandon et de réorientation durant les trois premières années demeurent élevés, les préoccupations politiques relatives à cette question à ce stade des études ne sont pas récentes. Nous citons à titre d'exemple la journée d'étude organisée par le Conseil National de la Jeunesse et de l'Avenir(1994) ${ }^{3}$ qui avait comme objectif principal de sortir avec des recommandations et des mesures permettant la prise en charge de cette problématique.

Eclairé, d'une part, par un tour d'horizon de la littérature existante sur ce sujet, ainsi que par les recommandations et suggestions de pratiques orientantes - formulées par les conférenciers et participants à la première édition de la Journée scientifique, organisée par nos soins, à l'université Ibn Tofail sur la thématique de l'orientation pédagogique et la problématique de l'insertion professionnelle des jeunes lauréats universitaires, d'autre part. Cet article ambitionne de mettre l'accent sur les défis auxquels se trouve confrontée l'université marocaine, à savoir l'accroissement des besoins globaux de formation et la nécessité de former pour l'emploi, mais aussi sur les lourdes contraintes qui pèsent sur elle, quant à l'accueil d'un public mal préparé pour la poursuite d'études supérieures. Ceci, dans l'optique de contribuer à la construction d'un cadre de réflexion sur les pratiques pédagogiques orientantes en introduisant un dispositif innovant nommé « Noviciat pédagogique ».

\section{L'orientation active à l'université marocaine :}

\section{L'orientation active : état des lieux}

L'orientation active a été approchée dans un premier temps au Québec (2001) par D. Pelletier ${ }^{4}$. Par la suite, elle a été reprise, en France, par D. Ferré 5 . Cette approche orientante place «le choix professionnel au cœur des apprentissages de l'apprenant. Elle lui permet de faire des liens entre ce qu'il apprend et son avenir, entre les matières enseignées et le monde du travail. Elle utilise les professions comme éléments de contextualisation des

${ }^{2}$ Information relatée par Mediltv.com : «Baccalauréat 2018 : un taux de réussite de 57,36\% » avec pour source l'Agence Marocaine de Presse (MAP), http://www.mediltv.ma/fr/baccalaur\%C3\%A9at-2018-un-taux-der\%C3\%A9ussite-de-57-36-actualit\%C3\%A9s-maroc-infos-121319

${ }^{3}$ Actes de la journée d'étude organisée par le Conseil National de la Jeunesse et de l'Avenir Rabat, 17 mai 1994

${ }^{4}$ Pelletier, D. (2004), L'approche orientante : la clé de la réussite scolaire et professionnelle, Sainte Foy (Québec), Septembre éditeur.

${ }^{5}$ Ferré. D., (2005), Pour une approche orientante de l'école française, Paris, Editions Qui plus est. 
apprentissages. C'est une nouvelle façon de contribuer à la réussite des élèves qui implique tous les membres de l'équipe éducative ». De ce fait, cette approche incite l'apprenant à être l'acteur de sa vie, de son devenir, et à se prendre en charge. Par ailleurs, l'approche orientante s'apparente aux principes de l'entrepreneuriat ${ }^{6}$.

Dans leur analyse, D. Pelletier et J.P. Boutinet ont souligné la dimension dynamique que revêt ce concept, prenant en compte toutes les transformations actuelles de la société et la place du sujet dans la complexité du temps présent. L'approche orientante cherche, par une meilleure compréhension de la complexité du rapport du sujet post-moderne à sa propre orientation, à permettre un meilleur accompagnement des apprenants, souvent déconnectés de toute temporalité. ${ }^{7}$ En effet, c'est dans le cadre de l'orientation et de l'insertion professionnelle que se détermineront les conduites des apprenants par rapport à un «Idéal du Moi », en relation avec le système scolaire et les conditions sociales de vie.

L'orientation peut être appréhendée également grâce aux apports de la psychanalyse ${ }^{8}$. Ainsi, les conseillers d'orientation et les psychologues travaillent avec le sujet sur les articulations à propos de l'« Identité/Sujet et Responsabilité/Praxis ${ }^{9}{ }^{2}$ en s'appuyant sur le paradigme psychanalyste qui considère l'identité comme un produit social trouvant sa source dans le regard des autres. Ils travaillent particulièrement sur les représentations professionnelles et sociales, la manière dont elles se mettent en place, leur mode de fonctionnement et leur évolution, et la manière dont chacun traite les informations en provenance de son environnement afin d'élaborer des conduites en cohérence avec ses projets personnels et de son avenir professionnel. Dans le contexte de l'éducation à l'orientation, le praticien de l'orientation s'apparente à un éducateur (Guichard, 1997) puisqu'il manifeste le même souci pédagogique que l'enseignant.

Néanmoins, il est important de rappeler que l'orientation croise des enjeux multiples. Ces derniers sont à la fois collectifs et individuels. Les premiers tentent de gérer au mieux les flux pour ajuster les apprenants au nombre de places dans les différents établissements de formation et aux débouchés prévus par les milieux économiques. Alors que les seconds (enjeux) s'inspirent de l'aspiration de chacun à réussir sa vie, et à développer ses compétences ; il s'agit pour chaque étudiant de trouver des études et un métier où il pourra s'épanouir, et pour ce faire il devra pouvoir bénéficier d'informations et de conseils.

Sans un accompagnement effectif de l'institution, certains facteurs externes peuvent agir comme freins à l'ambition. A ce titre, nous en citons la motivation et les représentations des élèves de leurs propres aptitudes, des parcours scolaires et des métiers, la pression exercée par les parents et les pairs, les contraintes socio-économiques du marché de l'emploi..., etc.

L'élève-étudiant arrive à l'université en portant en soi sa propre perception de l'université, celle imposée et/ou proposée par son environnement, ses ambitions, ses souhaits et ses contraintes. Il est tiraillé entre deux environnements. Le premier façonné par ses propres perceptions et représentations de son présent et de son devenir (les conditions socioéconomiques du marché de l'emploi) mais aussi par ses aptitudes intellectuelles et limites personnelles. Le second n'est autre que le produit de l'offre institutionnelle ou plus exactement de ce que l'université pourrait lui proposer ou lui imposer et qui se traduit par une réaction vis-à-vis des règlements institutionnels, une capacité d'adaptation et d'intégration, ou encore par l'influence plus ou moins partiale du corps enseignant, .... A l'ensemble des facteurs précités, s'ajoutent les résultats scolaires et l'éducation formelle à l'orientation qui facilitent ou au contraire entravent la réussite de l'orientation ${ }^{10}$ (Bowes et al. 2005).

Toutefois, une orientation informelle se nourrit de toute information fournie par la famille et/ou par les amis et surtout elle s'enrichit par leurs propres perceptions des études et de l'emploi. Nous pouvons dire qu'elle contribue

\footnotetext{
${ }^{6}$ Surlemont, B. et Kearney, P, Bruxelles, Pédagogie et esprit d'entreprendre. De Boeck, 2009

${ }^{7}$ Dubois, A. L'approche orientante, http://www.cahiers-pedagogiques.com/L-approche-orientante

${ }^{8}$ Francequin, G, (2002), Canadian Journal of Counselling / Revue canadienne de counseling, Vol. 36(2), 161-170, Psychologie de l'orientation, théorie de l'action et récits de vie.

9 Activité physiologique et principalement psychique, ordonnée à un résultat. FOULQ. St-Jean (1962), source: http://www.cnrtl.fr/definition/praxis

${ }^{10}$ Bowes. L, Smith, D \& S. Morgan (2005). Reviewing the evidence base for careers work in schools. Derby: Centre for guidance studies (CeGS), University of Derby.
} 
d'une manière ou d'une autre dans la préparation psychologique et même matérielle (le choix du parcours, la capacité à financer les études, ...) des étudiants. De ce fait, les jeunes ayant une idée claire de ce qu'ils veulent faire sont fréquemment influencés par un proche travaillant dans ce même secteur et auquel ils font confiance. Pour Y. Grelet $(2005)^{11}$, l'orientation vers des filières professionnelles n'est pas seulement conditionnée par les résultats scolaires : les aspirations des familles peuvent influencer les effets de l'origine sociale. En conséquence, « l'accès aux formations professionnelles se fait selon deux modèles extrêmes : celui de la transmission, propre aux indépendants (agriculteurs, artisans, commerçants) dont les enfants font le choix de la reproduction; et celui de la relégation, qui caractérise les orientations par défaut, motivées par l'échec scolaire. Entre les deux, la hiérarchie des spécialités se lit en miroir de la hiérarchie sociale. »

Certes, en l'absence d'une orientation officielle et formelle, amorcée par les institutions scolaires et universitaires, l' « orientation informelle » prend les devants et influence généralement le passage d'un cycle à un autre (Bowes et al. 2005).

Le Bastard-Landrier ${ }^{12}$ (2005) pense que la perception des élèves de leur niveau scolaire ou leur rapport aux disciplines, ont une incidence sur leurs résultats scolaires (en français et en mathématiques notamment) ainsi que la formulation de leur vœu d'orientation. Ceci dit, les choix d'orientation sont déterminés essentiellement par le sexe de l'élève, la catégorie socioprofessionnelle des parents et les résultats scolaires. L'expérience française montre également, grâce à l'étude initiée par E. Nauze-Fichet $(2005)^{13}$ que, sept ans après leur entrée au collège, une majorité de jeunes sont encore en formation initiale et déclarent avoir un projet professionnel, relevant ici un taux plus élevé auprès des apprentis, des élèves en terminale et, plus généralement, des filles. Ces résultats montrent l'existence d'influences croisées associant le parcours scolaire, le sexe et les caractéristiques du projet. Sans oublier également l'impact du coût, des résultats au baccalauréat, de la distance géographique ou des procédures d'inscription qui s'agencent aux autres facteurs.

En somme, l'orientation active se révèle être un processus social complexe, continu et inachevé, visant non seulement l'acquisition de compétences pour « construire sa vie ${ }^{14}$ (Guichard, 2005) mais également, d'une vision du monde plus élargie qui permet aux étudiants de «s'orienter dans la vie ${ }^{15}$ (Danvers, 2009). Cette dimension ne se limite pas aux impératifs exigés par la spécialisation universitaire, elle la dépasse pour atteindre la pluridisciplinarité.

\section{Pratique d'orientation en milieu universitaire}

«Une université, dans le cadre de son autonomie pédagogique, doit pouvoir examiner les dossiers des candidats et l'adéquation de leur profil avec les spécificités de la licence demandée. Elle doit pouvoir réorienter un candidat dont le projet personnel manque de cohérence. Plutôt que de «sélection », je préfère parler d'«"orientation active"». Dans cet esprit, une liste de prérequis devra bien entendu être élaborée par les universités pour chaque filière proposée. $\gg^{16}$

Telle était la position du ministère de l'enseignement supérieur marocain telle qu'exprimée par Monsieur le Ministre. Ce dernier a engagé, au titre de l'année 2018, de nouvelles réformes. Un tournant stratégique qui fait ressortir non seulement l'importance de l'orientation et de l'insertion professionnelle des étudiants qui doivent constituer une priorité pour l'université marocaine au même titre que la formation et la recherche scientifique. Mais

${ }^{11}$ Grelet, Y. (2005). «Enseignement professionnel, spécialité de formation et reproduction sociale ». Éducation et Formations, $n^{\circ} 72$.

${ }^{12}$ Le Bastard-Landrier, S. (2005). «L'expérience subjective des élèves de seconde : Influence sur les résultats scolaires et les vœux d'orientation ». L'orientation scolaire et professionnelle, vol. 34, n 2, p. 143-164.

${ }^{13}$ Nauze-Fichet, E. (2005). «Les projets professionnels des jeunes sept ans après leur entrée au collège ». Education et formations, no 72, p. 113-123.

${ }^{14}$ Guichard, J. (2005). L'orientation scolaire et professionnelle. Paris : Dunod.

${ }^{15}$ Danvers, F. (2009). S'orienter dans la vie : une valeur suprême ? Dictionnaire de sciences humaines. Villeneuve d'Ascq : Presses universitaires du Septentrion, $656 \mathrm{p}$.

${ }^{16}$ Entretien avec Monsieur le Ministre de l'Éducation nationale, de la Formation professionnelle, de l'Enseignement supérieur et de la Recherche scientifique, Edition $\mathrm{N}^{\circ}: 5260$ Le 26/04/2018, https://www.leconomiste.com/article/1027498-enseignement-vision-2030-les-grandes-nouveautes-du-ministre 
également, l'urgence d'une rationalisation de l'enseignement universitaire, par des filières aux exigences et aux débouchés clairement justifiés et identifiés, à instaurer et à développer pour anticiper et éviter les situations d'échec ou d'abandon universitaire.

Sous l'angle de l'orientation ou de l'approche orientante, l'université présente une particularité marquante, puisqu'elle constitue « un enseignement terminal qui, à la différence des systèmes qui le précèdent, ne prépare pas à l'accès à un autre système d'enseignement mais au contraire débouche, ou en tout cas est censé le faire, sur la vie professionnelle $\gg^{17}$ d'une part. Et d'autre part, elle s'identifie comme un espace de transition d'un « devenir élève » à un « devenir étudiant ». Selon A. Coulon ${ }^{18}$, le passage du statut d'élève au statut d'étudiant nécessite une véritable initiation, la découverte et l'appropriation d'« allants de soi », et l'acquisition d'une compétence. Les notions d'affiliation, de membre, de «praticalité » des règles permettent de décrire les mécanismes du processus de sélection et de classement qui distinguent ceux qui réussissent et ceux qui échouent.

C'est à la lumière de ces mutations que nous nous sommes intéressés à la question de l'orientation universitaire et plus précisément à ses différentes pratiques et à son impact sur l'insertion professionnelle de nos jeunes étudiants universitaires. Dans cette perspective, notre attention s'est portée sur la mise en place d'un système d'« orientation active » à l'université marocaine. Nous avons imaginé un dispositif d'orientation qui pourrait offrir une meilleure gestion des attentes personnelles et professionnelles de nos futurs diplômés.

\section{L'université marocaine et l'accueil des étudiants.}

L'université marocaine comme toutes les universités du monde s'efforce d'offrir une qualité d'enseignement supérieur digne des attentes de toutes les parties prenantes de son écosystème : étudiants, professeurs, recruteurs, société..., etc. Son souci ou sa mission qui, dans les faits, semblait confinée à celle de diplômer, semble enfin évoluer pour assumer désormais celle de former pour l'emploi.

L'emploi des jeunes diplômés, notamment celui des lauréats des établissements universitaires à accès ouvert, présente un épineux problème régulièrement signalé dans différents rapports et études tels que ceux du HautCommissariat au Plan marocain (HCP.ma). Le chômage de ces jeunes diplômés renseigne sur le déphasage existant entre la réalité du monde de l'emploi, les diplômes proposés par l'université marocaine et la perception qu'en ont les jeunes lauréats d'une part et les recruteurs d'autre part. Face à ce triste constat, l'intérêt que revêt notre problématique - de l'accueil des nouveaux étudiants à l'université et la nécessité de bien les préparer et les orienter pour renforcer leur aptitude à opérer un choix judicieux (de diplôme à débouchés professionnels) - prend tout son sens.

Certes, chaque année, l'université reçoit un nouveau public composé majoritairement de nouveaux bacheliers en sus d'un autre type de public qui, lui, aspire à l'amélioration de son quotidien (par une évolution de son statut professionnel) en investissant davantage dans la formation pour améliorer sa situation financière (pour ceux qui sont en poste) ou tout simplement pour consolider ses connaissances et/ou son statut social. Ce nouveau public s'attend apriori à un accueil individuel et à une écoute personnalisée. Et donc, à un accompagnement adapté dans ce nouvel univers susceptible de lui permettre une initiation appropriée dans ce monde universitaire. Le passage du lycée à l'université est en réalité déroutant, voire déstabilisant, pour ces « élèves » de longue date, promus soudainement au rang d'étudiants, à la fois pour des raisons matérielles (espace nouveau, autonomie nouvelle et non-directivité de l'environnement) mais aussi du fait que l'université est souvent vue et vécue comme un choix par défaut quand elle n'est pas une solution temporaire dans l'attente d'une réintégration dans une filière sélective. L'université reçoit donc des publics souvent peu déterminés et peu informés sur leurs possibilités d'avenir. Un certain nombre d'entre eux s'oriente par défaut vers des disciplines nouvelles, qui leurs sont méconnues et dont ils ne savent rien ou presque rien et dans lesquelles, le plus souvent, ils peuvent échouer massivement.

Un ensemble de propositions et de limites ont été formulées et soulignées lors de la première édition de la journée scientifique OFIP-1 (Orientation pédagogique, Formation et Insertion professionnelle : « relever le défi de la réussite ", organisée par nos soins et qui s'est tenue sur le campus universitaire de Kénitra, Maroc (Université Ibn Tofail), le 15 Mai 2018.

\footnotetext{
${ }^{17}$ Bireaud, A. (1993). Les méthodes pédagogiques dans l'enseignement supérieur (compte-rendu)

${ }^{18}$ Coulon, A. (1997), Le métier d'étudiant. L'entrée dans la vie universitaire Paris : PUF. - 219 p. ; (Politique d'aujourd'hui).
} 
En matière d'orientation, l'offre de l'université demeure insuffisante et incomplète. Aussi, parmi les points soulignés lors de cette manifestation, figurent ceux relatifs à la quasi-absence de contrôle(s) et de suivi de l'offre de formation et à la pléthore de formations sans débouchés professionnels réels ou viables.

Les nouveaux étudiants s'inscrivent dans des formations sans en saisir l'intérêt, ni pouvoir se renseigner sur leurs débouchés, encore moins sur la valeur que leur accorde le marché du travail, ou sur les compétences requises pour les réussir ou même sur celles à développer en cours de formation.

Cette initiation et préparation ne peuvent se faire sans la participation et la collaboration effectives des responsables de l'orientation et du cycle secondaire et de ceux de l'enseignement supérieur.

Or, l'action de l'orientation (pédagogique) voit son système marqué par un hiatus et ceci dès l'amont du processus, puisque déjà à l'échelle du cycle secondaire, et sans parler de toutes les lacunes dont souffre ce système d'orientation, il suffit de citer les quelques faits saillants suivants : un (1) conseiller pour quatre-mille (4000) élèves, l'omission ou l'exclusion de certaines zones géographiques défavorisées, le problème d'accès à l'information, ... etc. D'où l'intérêt de la mise en place d'une véritable stratégie en matière d'orientation. Et, qui soit cohérente et concertée entre les spécialistes de l'enseignement et de l'orientation des deux cycles, pour synchroniser avec l'offre de formation réelle de l'université, mais aussi qu'elle prépare "l'ancien élève \& jeune-étudiant» aux divers changements qu'il va connaitre : un nouvel espace ou univers (qu'est celui du monde universitaire), nouvelles méthodes d'enseignement, souvent radicalement différentes d'un niveau à l'autre dans les mêmes disciplines, un nouveau rythme de vie (étudiante), de nouveaux enseignants (qui eux solliciteront la contribution de ce nouveau étudiant à sa propre formation et viseront à le rendre progressivement autonome), ... etc.

Dans ce sens, il importe de souligner que l'université marocaine est consciente, depuis belle lurette, de l'intérêt d'un accueil adéquat des nouveaux bacheliers et de celui d'une orientation adaptée de ces derniers. Il convient de signaler donc qu'un certain nombre d'initiatives ou de dispositifs d'orientation existent. A titre d'exemple, le centre d'accueil, d'information, d'orientation et de suivi (CAIOS) ${ }^{19}$ mis en place, il y a plus d'une décennie, par l'université Mohamed V de Rabat (depuis 2005) au profit des jeunes étudiants et des bacheliers. Une expérience parmi d'autres qui restent insuffisantes à l'aune des attentes des étudiants dont l'effectif ne fait que grimper.

Une vision stratégique en matière d'orientation exige nécessairement une concertation à la fois entre les deux cycles (secondaire et supérieur) et entre les acteurs de ces derniers et ceux du monde socioprofessionnel (une ouverture sur l'écosystème socioéconomique, ses besoins et ses exigences). Une politique d'ensemble s'avère donc primordiale pour espérer atteindre les objectifs escomptés. Pour ce faire, c'est la mise en place, non seulement, d'un service d'information, d'accueil et d'orientation, en charge de distribuer des brochures et des dépliants, mais aussi et surtout d'une structure -à l'image du $1^{\text {er }}$ réseau d'accueil et d'information des jeunes en France, constitué de 1530 structures (dont les $\mathrm{CRIJ}^{20}$, CIDJ, ... etc.) maillant tout le territoire français et qui accueille environ 5 millions de jeunes chaque année en France, par exemple- ou d'une cellule « de veille et de coordination » ayant pour mission de produire des études statistiques et de recueillir des données stratégiques (compétences demandées, secteurs porteurs, états des lieux du marché de l'emploi, son évolution, ses mutations et tendances, une information et des mises à jour juridiques sur le code du travail, ... etc.) et qui soit en charge aussi bien de sonder et d'analyser les besoins du public et ses attentes (en dépit de son hétérogénéité) que d'évaluer et de suivre l'évolution de l'offre de formation et son degré d'adéquation aux attentes de ces publics (dont celui des recruteurs). Ceci, passe obligatoirement par la participation de la cellule (ou de la structure) suggérée à l'ingénierie de formation, surtout dans la mesure où il serait possible de la faire bénéficier des appuis nécessaires, des moyens et/ou du soutien des responsables administratifs, du corps enseignant et pourquoi pas de celui du monde de l'entreprise qu'il faudrait associer à cette démarche, pour lui permettre ainsi d'agir, en amont, sur la qualité (ou les qualités) de ses futurs salariés ou collaborateurs.

\footnotetext{
${ }^{19}$ Centre d'Accueil d'Information, d'Orientation et de Suivi (CAIOS), crée en 2005. http://caios.um5.ac.ma/

${ }^{20}$ Les CRIJ (Centre Régional Information Jeunesse), CIDJ (Centre d'information et de documentation jeunesse), sont des structures relevant du réseau IJ (Information jeunesse) qui est un réseau (français) remontant à 1969 et issu des mouvements de l'éducation populaire (en France), ses structures existent sous forme associative financés par l'État et les collectivités territoriales, entre autres. https://www.orientation-pour-tous.fr/etudier-seformer/rechercher-une-adresse-utile/Centre-Regional-Information
} 
Dans cet article, nous avons l'ambition d'apporter ou de proposer un regard nouveau sur la pratique de l'orientation pédagogique. Cette proposition, que nous considérons innovante, est une piste à explorer par les instances pédagogiques et institutionnelles. Une vision novatrice qui s'appuie sur l'idée selon laquelle le savoir enseigné est construit collectivement (approche socioconstructiviste) et la manière dont fonctionnent respectivement les processus d'évaluation, de sélection et d'exclusion, lesquels montrent la place importante qu'occupent les enseignants et les spécialistes d'orientation dans l'ensemble du processus d'accueil et d'orientation.

\section{Une pratique d'orientation innovante : « Noviciat pédagogique »}

Le développement des pratiques éducatives en orientation s'explique à la fois par le référent idéologique qui prêche l'importance de la construction de soi et le développement personnel, perçus comme clés du progrès économique et social. Mais aussi par l'évolution de l'emploi et la problématique de l'insertion professionnelle. Selon cette conception, il semble opportun de préparer chacun à faire face aux multiples transitions auxquelles il sera confronté tout au long de sa vie (Guichard, 2000) ${ }^{21}$. Outre cette double évolution, celle de la massification a conduit, quant-àelle, à ce que l'accent soit mis davantage sur le mécanisme de gestion des flux, ou plutôt sur les modalités de répartition des flux d'élèves ou d'étudiants, en mettant à leur disposition, en sus, ou en lieu et place, des séances d'orientation ou de conseils usuels (correspondant à un ensemble de cas prédéfinis), une liste de critères et outils susceptibles de mieux leur permettre l'appréciation de la viabilité de leurs choix (ou celle des alternatives d'orientation académico-professionnelle qui s'offrent à eux) et delà la perception d'une trajectoire personnelle de spécialisation professionnelle de manière plus ou moins réaliste.

Une responsabilisation qui marque cette transition qu'est le passage du statut d'élève à celui d'étudiant (universitaire).

\section{Le « Noviciat pédagogique » comme dispositif innovant d'orientation}

Notion -dérivée d'un mot d'origine religieuse (biblique) - qui signifie « mouvement engageant toute la vie affective et intellectuelle, qui porte quelqu'un à choisir une certaine profession $»^{22}$. Ce concept est défini également comme une préparation des novices à la vie religieuse, qui consiste à éprouver leur vocation et dont la durée varie selon l'ordre, la congrégation ; état des novices pendant cette formation ${ }^{23}, \ldots$ etc.

A l'examen des définitions susmentionnées, nous avons été interpelés par la similitude ou l'analogie possible entre la notion de noviciat religieux et celui pédagogique qui est au coeur de notre problématique. Nous avons décelé dans ces significations les prémices d'un dispositif et/ou approche originale pour traiter cet aspect crucial du passage «Lycée-Université » dans la vie de l'«élève-étudiant ». Du fait que cette phase préparatoire et d'initiation, ou période probatoire à l'engagement dans la vie (fût-elle religieuse ou professionnelle), correspond dans les deux cas à un changement de statut, de vocation, de profession, de rythme de vie, de communauté et de « devenir ». Nous pensons donc que cette délicate transition du statut d'élève à celui d'étudiant mérite définitivement une consécration par ce vocable de «Noviciat pédagogique » que nous proposons ici pour immortaliser et célébrer ce moment de la vie de l'《apprenant-collaborateur »-dans le contexte tant de sa formation universitaire que de celui de son parcours professionnel- en devenir, comme une phase critique et décisive qui mérite toute l'attention eu égard à ses enjeux.

Notre tour d'horizon des pratiques d'orientation mises en place dans la dizaine d'universités que compte notre pays, nous a révélé que la pratique d'orientation au Maroc était véritablement bornée dans le temps (occasionnelle, limitée à quelques jours et à quelques brochures sans ou avec conseils génériques) et que le sujet en question (l'élèveétudiant) se trouvait désemparé face à un ensemble d'exigences : respects des délais -souvent contraignantsd'inscription, de réinscription et ceux du calendrier de début des études et celui des examens ou tests d'accès, la capacité d'accueil de la filière visée, ... etc. Ce qui rend les conditions de prise de décision déplorables pour l'étudiant-bachelier, généralement perdu et isolé. Et qui se voit donc, le plus souvent, contraint de prendre en charge, tout seul, la lourdeur et de cette démarche et des éventuelles conséquences fâcheuses de ses choix. Nos propos peuvent être illustrés ici par si ce n'est qu'un exemple (flagrant) qui nous a assez marqué lors des témoignages des étudiants ayant pris part à la première édition de la journée OFIP-1 à savoir celui d'une néo-bachelière qui a choisi

\footnotetext{
${ }^{21}$ Guichard, J. (2000). Quelles peuvent être les finalités individuelles et collectives de l'éducation en orientation ? Paris : Inetop

${ }^{22}$ Grand Larousse de la langue française, 1989.

${ }^{23}$ Dictionnaire du Centre National de Ressources Textuelles et Lexicales : www.cnrtl.fr/definition/noviciat
} 
de "s'aventurer" dans une filière tout simplement parce que la file d'attente pour le dépôt de dossier d'inscription à cette dernière était, comparativement aux autres, des moins animées et ceci pour pouvoir rentrer chez-elle (à 150 bornes delà) à temps (c'est-à-dire avant le coucher du soleil) en l'absence du toit d'un proche pour l'accueillir et l'abriter sur la ville de son inscription.

Nous soulignons également, que le pont liant l'université au monde de l'entreprise et à son écosystème supposé assurer le passage fluide et sans à-coups de ce jeune chercheur d'information(s), de conseils ou de stage de la rive des connaissances essentiellement académiques ou théoriques à celles de leur expérimentation et mise en pratique, se trouve malheureusement suspendu. Les raisons de la rupture entre ces deux mondes sont multiples et souvent difficiles à déterminer, voire même à expliquer. A titre indicatif, nous signalons celles relatives aux difficultés de gestion de "la masse" d'un côté et de l'autre, celles des innombrables demandes (reçues) du côté des acteurs socioéconomiques et celles des encadrements et du suivi (à assurer) de la part de l'université. Sans pouvoir être exhaustif, on peut également évoquer le problème que rencontre l'officialisation des conventions signées entre les universités et les professionnels et supposées assurer le rapprochement des étudiants du monde de l'emploi.

\section{Pourquoi le noviciat pédagogique?}

L'orientation pédagogique, qu'elle soit en amont ou en aval, des étudiants s'inscrit au coeur de l'action pédagogique et joue un rôle fondamental dans l'atteinte des attentes des jeunes en termes de formation, d'intégration et d'insertion professionnelle.

Nul n'ignore, qu'au cours de leur formation jusqu'à la sortie de l'université pour affronter la vie professionnelle, ces jeunes ont besoin d'être aidés, soutenus par des conseils, informés des changements survenus dans le monde du travail et encouragés dans le choix de leurs carrières.

Pour lutter contre le taux d'échec important des étudiants en première année, l'université est invitée à mettre en place un dispositif d'accompagnement, d'orientation et de réorientation pédagogique plus large dont l'objectif se doit de dépasser, et de loin, la simple information pour s'élargir et inclure celui de l'accompagnement méthodologique. Ce dernier prenant la forme d'une mise à niveau tant des connaissances fondamentales en langue, en techniques d'expression et de communication adaptées au contexte professionnel, par des formateurs bidisciplinaires, qu'en développement des méthodes de travail en groupe, en culture d'entreprise, entrepreneuriat, leadership, en développement personnel, gain d'autonomie, et par une aide à l'orientation et à la construction d'un projet personnel et professionnel, mais aussi un projet de formation.

Ce «noviciat pédagogique » n'est autre qu'un temps de préparation et de réflexion pour choisir la bonne orientation et permettre ou offrir ainsi, à l'élève-étudiant, la possibilité de réussir un parcours universitaire (en connaissance de cause, pour une meilleure réussite, par plus de motivation et de conviction), grâce à une remise à niveau mais aussi une préparation pédagogique et méthodique à l'environnement universitaire en s'initiant aux nouvelles méthodes de travail, d'organisation et d'apprentissage. Ce noviciat pédagogique exige une réelle coordination (effective) à l'échelle de l'université mais également au niveau des académies régionales puisque la question de la réussite, comme celle de l'échec ou de l'abandon universitaire, notamment en première année, intéressent tous les niveaux et toutes les filières de l'enseignement.

L'échec ou l'abandon universitaire s'explique par des méthodes de travail non adaptées à l'enseignement supérieur ou encore par un manque de motivation lié à une orientation mal définie. Pour contrecarrer cet échec précoce dans l'enseignement supérieur, l'université est appelée à agir, à prendre des mesures, à engager des actions et à développer les compétences transversales nécessaires en mettant en place un véritable projet de formation.

Pour la réussite de ce dispositif, il doit être pensé et déployé par une équipe pluridisciplinaire associant et des enseignants du second cycle et du supérieur, ainsi que des spécialistes de l'orientation, le tout doit bénéficier d'un appui inconditionnel des responsables administratifs. Ce cursus se déroulerait idéalement à compter du mois de mars et jusqu'au mois d'août. Et, se composerait d'une série d'activités et d'ateliers de formation et d'accompagnement qui permettraient au nouveau bachelier tant de mieux découvrir les options académiques et réalités professionnelles, que de s'outiller de compétences et de se préparer (pédagogiquement) pour assumer leur nouveau statut d' « étudiants universitaires». 
Le Noviciat pédagogique ne concerne pas seulement les novices, mais aussi celles et ceux ayant entrepris des études supérieures mais n'ayant pas foncièrement réussi leur semestre ou n'ayant pas apprécié le (ou leur) choix de formation. L'occasion de (re)découvrir, pendant ce laps de temps, l'offre pédagogique et de formation de l'université, en tant qu'alternative, pour ce public "égaré", voir potentiellement "perdu", lequel en son absence se verrait vraisemblablement vouer au décrochage. Ainsi, l'ouverture sur des enseignements disciplinaires et l'accompagnement via des entretiens individuels révéleront assurément de nouvelles pistes de poursuite des études. Ces entretiens individuels permettront de repenser les compétences acquises, ou à acquérir, que l'étudiant devra travailler ou sera amené à retravailler autrement pour réussir sa nouvelle formation. En outre, plusieurs actions « surmesure » existent, et peuvent être mises en place, notamment celles ciblant la « re-motivation » des étudiants surtout ceux qui se sont retrouvés à l'université, par défaut, de par leur incapacité à réussir les concours d'accès aux grandes écoles.

L'originalité de ce dispositif (de "noviciat pédagogique") ne réside pas ou ne se limite pas à la seule et unique mission d'accueil et d'orientation (déjà assurée par les structures classiques d'information) mais dans son approche synergique nouvelle impliquant davantage l'apprenant, le corps enseignant et le ou les partenaire(s) socioprofessionnel(s). Une démarche qui nécessite que l'organisation des activités et des actions à mener soit planifiée préalablement à l'arrivée du nouveau bachelier à l'université.

A partir du mois de mars, l'élève futur étudiant, doit être sensibilisé et invité à assister à des séances (sur place -au lycée- ou à l'université) d'information et d'échange autour de diverses thématiques liées au projet personnel et professionnel, au métier d'avenir, aux compétences transversales et comportementales à développer, au marché de l'emploi, à la méthodologie du travail universitaire et aux nouvelles pratiques enseignantes (pédagogie active, enseignement à distance, espace de travail numérique, étude de terrain, approche des professionnels, recherche d'information et de conseils sur le terrain, etc.)

Il est à rappeler que l'orientation active nécessite un traitement particulier et individuel des voeux des élèves sur la base d'un dossier (composé de formulaires et de pièces-jointes) examiné par des commissions en charge d'étudier ces souhaits en fonction de la capacité des candidats à suivre tel ou tel cursus universitaire au moins jusqu'à la licence, mais aussi selon le degré d'exigence de la filière envisagée et de ses débouchés.

Ainsi, le processus d'orientation regroupe trois actions corrélées, se présentant comme suit :

1. action informationnelle : le jeune bachelier reçoit toute l'information nécessaire concernant les différentes formations proposées et les métiers d'avenir, la situation du marché de l'emploi. Et ceci, par le biais de supports de communication variés (papier et/ou numérique). A titre d'exemple, par le biais d'une plateforme (ou site web) dédiée qui le renseignerait sur l'offre de formation ainsi que sur les taux de réussite et d'insertion professionnelle.

2. action d'orientation : le jeune bachelier ou même l'étudiant ayant fait un mauvais choix bénéficie d'un entretien individuel par des équipes mixtes (spécialistes d'orientation, responsables pédagogiques, enseignants et représentant des secteurs professionnels), avec la possibilité d'être accompagné dans sa prise de décision.

3. action pédagogique : le jeune bachelier est invité à suivre un programme de préparation à l'entrée en formation et qui pourrait aller, selon son besoin, d'une remise à niveau (linguistique ou disciplinaire), en passant par une aide à la préparation du projet personnel et professionnel, et/ou à la découverte professionnelle (visite d'entreprises et rencontre avec les professionnels), et jusqu'à se voir proposer des ateliers animés par les professeurs sur les nouvelles pratiques enseignantes universitaires (études de cas, pédagogies de groupe et de projet, enseignement et accompagnement ou suivi à distance) et d'autres sur la méthodologie du travail universitaire.

L'enseignement du Projet Professionnel de l'Étudiant (PPE), évoqué au niveau de cette troisième action, par une équipe de professeurs bien formée, engagée et impliquée pour garantir un encadrement pédagogique de qualité et récolter les résultats escomptés, permettra certainement la réussite de ce dispositif.

Destiné à assurer une continuité entre l'enseignement secondaire et l'enseignement supérieur, la réalisation de ce dispositif (de noviciat pédagogique) implique nécessairement une organisation, par niveau, d'un calendrier d'activités préparant et accompagnant les élèves du lycée vers l'université. La réussite du Noviciat pédagogique 
nécessite donc un engagement réel non seulement de l'université ou du lycée mais très certainement d'autres institutions publiques et privées en vue de médiatiser les passerelles existantes et permettre de nouvelles entre les formations au profit des étudiants qui ont raté leur orientation, et sont en réorientation, mais aussi pour favoriser les collaborations pédagogiques entre enseignants des deux systèmes d'enseignement (secondaire et universitaire).

Des actions complémentaires peuvent être mises en place, tels que des forums, des actions ou sessions d'immersion et d'accueil, rencontres entre pairs, intervention d'enseignants du supérieur auprès des élèves du secondaire, des actions d'appui mobilisant des spécialistes (équipes mixtes des deux systèmes, et des mondes académiques et professionnels) auprès des étudiants pour les aider à étayer leurs parcours individuels et finaliser l'expression de leurs choix.

\section{Conclusion:-}

Pour faire face à cet impératif politique, économique et social qu'est la formation de citoyens aptes à s'insérer de manière durable sur le marché du travail, à prendre part activement aux développement de l'économie nationale, à contribuer à sa compétitivité et à relever ainsi les défis sociétaux et internationaux, tels que ceux relatifs au développement humain et à la prospérité de la nation, etc, un ensemble d'acteurs « éducatifs » sont particulièrement désignés, sollicités pour et concernés par ces évolutions et ces défis. Au premier rang figure évidemment « l'université », qui par la place stratégique qu'elle occupe dans la sphère de l'enseignement, [...] puisqu'elle abrite « un enseignement terminal qui, à la différence des systèmes qui le précèdent, ne prépare pas à l'accès à un autre système d'enseignement mais au contraire débouche, ou en tout cas est censé le faire, sur la vie professionnelle » (Coulon, 1993).

Aussi, l'université se doit de jouer son rôle de stimulateur eu égard aux voeux d'orientation qu'elle reçoit et qu'elle doit s'efforcer de satisfaire autant que faire se peut. Ceci, tenant compte notamment de la singularité du public qu'elle accueille, et qui est composé majoritairement de néo-bacheliers, pleins d'espoir, et qui aspirent à un avenir professionnel meilleur via une filière donnée. Hélas, ce n'est pas toujours le cas, faute d'une organisation appropriée et de moyens adaptés aux effectifs conséquents qui affluent vers elle. En sommes, les modestes mesures qu'engage l'université marocaine pour favoriser la prise en compte des souhaits de cette jeunesse en termes de choix et de qualité de formation, d'aide à la réussite et de professionnalisation des formations laissent à désirer.

L'orientation à l'université devrait cesser de se faire par défaut, pour s'opérer plutôt, dans le cadre d'une démarche positive, éclairée et réellement accompagnée.

Notre étude de la problématique de l'accueil et de l'orientation des nouveaux étudiants dans les universités marocaines et de la réorientation des étudiants en échec en fin de première année qui a fait l'objet du présent article a couvert l'examen des pratiques d'orientation à l'université marocaine qui a révélé plusieurs limites au niveau de l'approche orientante. La plupart des structures d'orientation et d'information mises en place œuvrent à la diffusion de l'information sans pour autant répondre aux demandes précises des étudiants et sans que ces derniers ne soient réellement impliqués. Ce qui réduit leurs chances de succès à l'université et leur possibilité de réussir brillamment leur formation et intégration du marché du travail.

Ce pourquoi, l'université devrait clarifier son offre de formation, assurer le suivi de ses étudiants et permettre une meilleure lisibilité en termes de « retour sur investissement ${ }^{24}$ des parcours qu'elle propose (Romainville, 2000). Et, est appelée à créer « régulièrement de nouveaux domaines professionnels, de nouveaux types de professionnels, de nouvelles compétences professionnelles » ${ }^{25}$ (Agulhon \& al. 2012)

Les recommandations proposées et conclusions formulées par les participants aux actes de la journée scientifique de l'orientation pédagogique et de l'insertion professionnelle OFIP-1 peuvent être ramenées aux trois principaux axes suivant :

1. L'orientation et l'insertion professionnelle des étudiants doivent être une priorité pour l'université marocaine au même titre que la formation et la recherche scientifique ;

2. L’offre d'enseignement universitaire doit cadrer avec ses débouchés ;

\footnotetext{
${ }^{24}$ Romainville, M. (2000). L'échec dans l'université de masse. Paris : L'Harmattan.

${ }^{25}$ Agulhon, C., Convert, B., Jakubowski, S., \& Gugenheim, F. (2012). La professionnalisation : Pour une université « utile »? Paris : L'Harmattan.
} 
3. Des structures d'orientation, d'accompagnement et de suivi dans les établissements universitaires doivent être créées.

La réalisation de ces objectifs ne se fera que par la mobilisation de l'ensemble des acteurs afin d'identifier, recenser et accompagner les étudiants qui optent pour la formation universitaire (notamment auprès des établissements à accès ouvert) et ceux qui, en cours de route, ne l'ont pas réussie. Aussi, et en vue de l'atteinte de ces objectifs, nous préconisons la mise en place d'une politique volontaire ou volontariste de la part de tous les acteurs universitaires, conjuguée à une rigueur administrative se traduisant par un engagement (effectif) de mise à disposition de tous les moyens et la logistique nécessaire pour motiver et pérenniser l'enthousiasme des parties prenantes, susciter l'implication d'autres parties et répandre ainsi par cet appui les valeurs de volontarisme et/ou volontariat. De plus, la mise en oeuvre de cette stratégie doit s'appuyer sur une bonne politique de communication pour faire connaître les outils nouvellement développés et les nouveaux services et activités à mettre en place.

Le présent article a cherché à examiner l'orientation pédagogique et son impact sur la réussite académique et professionnelle des étudiants en faisant de l'accompagnement pédagogique un levier de réussite des étudiants, un processus susceptible d'intéresser et de bénéficier en particulier à ceux d'entre eux qui visent à gagner en autonomie (universitaire) en identifiant ou en se fixant un but professionnel plus ou moins clair à atteindre.

En s'inspirant de l'approche orientante, le noviciat pédagogique se veut une pratique innovante de l'orientation à l'université marocaine. Cet accompagnement personnalisé qui correspond à une période de préparation visant à amener l'élève bachelier et nouvel étudiant à construire progressivement son parcours de formation, qui soit réfléchi et fondé sur l'orientation active, la préparation à l'enseignement supérieur et la découverte du marché de l'emploi. Il s'inscrit donc dans une démarche de pratique réflexive où la réflexion et l'analyse des pratiques mènent au développement de compétences chez l'étudiant néo-bachelier. Ce nouveau dispositif qu'est le noviciat pédagogique ne prétend pas fournir une réponse unique à la question de l'orientation et de l'accompagnement pédagogique mais se propose d'être un point d'appui à d'autres études qui vont enrichir certainement la réflexion sur ce sujet.

\section{Bibliographie: -}

1. Article de presse par Driss LYAKOUBI, paru dans le quotidien francophone $\mathrm{n}^{\circ} 1$ au Maroc, le journal Le Matin, du 18/05/2018, et intitulé : «L'orientation pédagogique, une absence aux conséquences désastreuses », URL: https://lematin.ma/journal/2018/lorientation-pedagogique-absence-aux-consequences-desastreuses/293063.html

2. Information relatée par Medi1tv.com : «Baccalauréat 2018 : un taux de réussite de 57,36\% » avec pour source l'Agence Marocaine de Presse (MAP), http://www.mediltv.ma/fr/baccalaur\%C3\%A9at-2018-un-taux-der\%C3\%A9ussite-de-57-36-actualit\%C3\%A9s-maroc-infos-121319

3. Actes de la journée d'étude organisée par le Conseil National de la Jeunesse et de l'Avenir Rabat, 17 mai 1994

4. PELLETIER, D., (2004), L'approche orientante : la clé de la réussite scolaire et professionnelle, Sainte Foy (Québec), Septembre éditeur.

5. FERRE. D., (2005), Pour une approche orientante de l'école française, Paris, Editions Qui plus est.

6. SURLEMONT, B. et KEARNEY, P, Bruxelles, Pédagogie et esprit d'entreprendre. De Boeck, 2009

7. DUBOIS, A. L'approche orientante, http://www.cahiers-pedagogiques.com/L-approche-orientante

8. FRANCEQUIN, G, (2002), Canadian Journal of Counselling / Revue canadienne de counseling, Vol. 36(2), 161-170, Psychologie de l'orientation, théorie de l'action et récits de vie.

9. Activité physiologique et principalement psychique, ordonnée à un résultat. FOULQ. St-Jean (1962), source : http://www.cnrtl.fr/definition/praxis

10. BOWES. L, Smith, D \& S. Morgan (2005). Reviewing the evidence base for careers work in schools. Derby: Centre for guidance studies (CeGS), University of Derby.

11. GRELET, Y. (2005). «Enseignement professionnel, spécialité de formation et reproduction sociale ». Éducation et Formations, $n^{\circ} 72$.

12. LE BASTARD-LANDRIER, S. (2005). «L'expérience subjective des élèves de seconde : Influence sur les résultats scolaires et les vœux d'orientation ». L'orientation scolaire et professionnelle, vol. $34, \mathrm{n}^{\circ} 2$, p. 143164.

13. NAUZE-FICHET, E. (2005). «Les projets professionnels des jeunes sept ans après leur entrée au collège ». Education et formations, no 72, p. 113-123.

14. GUICHARD, J. (2005). L'orientation scolaire et professionnelle. Paris : Dunod. 
15. DANVERS, F. (2009). S'orienter dans la vie : une valeur suprême ? Dictionnaire de sciences humaines. Villeneuve d'Ascq : Presses universitaires du Septentrion, $656 \mathrm{p}$.

16. Entretien avec Monsieur le Ministre de l'Éducation nationale, de la Formation professionnelle, de l'Enseignement supérieur et de la Recherche scientifique, Edition $\mathrm{N}^{\circ}: 5260$ Le 26/04/2018, https://www.leconomiste.com/article/1027498-enseignement-vision-2030-les-grandes-nouveautes-du-ministre

17. BIREAUD, A. (1993). Les méthodes pédagogiques dans l'enseignement supérieur (compte-rendu)

18. COULON, A. (1997), Le métier d'étudiant. L'entrée dans la vie universitaire Paris : PUF. - 219 p. ; (Politique d'aujourd'hui).

19. Centre d'Accueil d'Information, d'Orientation et de Suivi (CAIOS), crée en 2005. http://caios.um5.ac.ma

20. Centre Les CRIJ (Centre Régional Information Jeunesse), CIDJ (Centre d'information et de documentation jeunesse), sont des structures relevant du réseau IJ (Information jeunesse) qui est un réseau (français) remontant à 1969 et issu des mouvements de l'éducation populaire (en France), ses structures existent sous forme associative financés par l'État et les collectivités territoriales, entre autres. https://www.orientation-pourtous.fr/etudier-se-former/rechercher-une-adresse-utile/Centre-Regional-Information

21. GUICHARD, J. (2000). Quelles peuvent être les finalités individuelles et collectives de l'éducation en orientation? Paris : Inetop

22. Grand Larousse de la langue française, 1989

23. Dictionnaire du Centre National de Ressources Textuelles et Lexicales : www.cnrtl.fr/definition/noviciat

24. ROMAINVILLE, M. (2000). L'échec dans l'université de masse. Paris : L'Harmattan.

25. AGUlhon, C., Convert, B., Jakubowski, S., \& Gugenheim, F. (2012). La professionnalisation : Pour une université « utile»? Paris : L'Harmattan. 